\title{
Motor Neuron Disease and Frontotemporal Lobar Degeneration: A Tale of Two Disorders Linked To TDP-43
}

\author{
Lauren B. Elman Leo McCluskey Murray Grossman \\ Department of Neurology, University of Pennsylvania School of Medicine, Philadelphia, Pa., USA
}

\section{Key Words}

Motor neuron disease - Amyotrophic lateral sclerosis •

Frontotemporal lobar dementia

\begin{abstract}
Motor neuron disease (MND) is a neurodegenerative condition long thought to be associated only with motor weakness. Recent work now shows that cognitive difficulties are present in up to half of the patients with this disorder. About 5-10\% of patients with MND have a frank dementia that resembles frontotemporal lobar degeneration (FTLD). Imaging studies show quantitative abnormalities that resemble FTLD. Moreover, biochemical studies of ubiquinated histopathologic abnormalities in MND and FTLD reveal identical inclusions of TDP-43. These findings underline a fundamental link between MND and FTLD. This paper reviews this body of work.

Copyright $\odot 2008$ S. Karger AG, Base
\end{abstract}

\section{Introduction}

Motor neuron disease (MND) is a neurodegenerative disorder with clinical manifestations long thought to be limited to the pyramidal motor system. Amyotrophic lateral sclerosis (ALS) is the prototypical form of MND and is clinically manifested as the combination of signs and symptoms due to degeneration of upper motor neurons (UMNs) in the motor cortex and lower motor neurons
(LMNs) in the brainstem and spinal cord. Increasingly, clinical, imaging and neuropathological data are accumulating that redefine MND as a multisystem neurodegenerative disease. Cognitive abnormalities now appear to be the most common nonmotor finding in MND. In this paper, we describe MND and some of the associated cognitive impairments. We also consider biomarkers and etiological factors that link MND to another neurodegenerative condition, frontotemporal lobar degeneration (FTLD).

\section{Clinical Characteristics of MND}

The most common feature of MND is a disorder of voluntary motor functioning. MND refers to a group of progressive neurodegenerative disorders classically characterized by the degeneration of UMNs and/or LMNs responsible for the control of all voluntary skeletal muscle [1-5]. The cell bodies of the UMNs are located in the frontal lobes of the brain. Their axons traverse the white matter of the cerebral hemispheres, the brainstem and the spinal cord to project on to LMNs located in the brainstem motor nuclei and the gray matter of the spinal cord. The axons of these LMNs in turn project to target muscles in cranial, cervical, thoracic or lumbosacral segments of the body. Normal control of any voluntary skeletal muscle requires both intact UMN and LMN segments, and voluntary muscular function is compromised and may even be completely lost without both of these com-

\section{KARGER \\ Fax +4161306 1234 E-Mail karger@karger.ch} www.karger.com

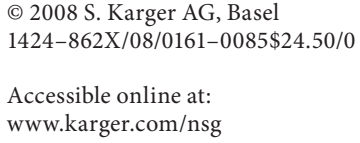

Dr. Lauren Elman

Department of Neurology, Pennsylvania Hospital

330 S. 9 th Street

Philadelphia, PA 19107 (USA)

Tel. +1 215829 7512, Fax +1 215829 6606,E-Mail elmanl@uphs.upenn.edu 
ponents. Thus, the cardinal symptom of MND is progressive muscle weakness. This includes the muscles responsible for breathing and swallowing, and as a result, MND is a life-threatening group of disorders.

At present there is no definitive diagnostic laboratory test for MND. MND is defined clinically on the basis of the neurological history, neurological examination and laboratory testing, including neuroimaging and electrophysiological studies, used to exclude disorders that may mimic MND. UMN loss is characterized by mild muscle weakness accompanied by stiffness, slowness of movement, dyscoordination, limb spasticity, hyperactive tendon reflexes and pathological reflexes. LMN loss is characterized by more severe muscle weakness with accompanying atrophy of involved muscles, muscular twitching (fasciculations) and hyporeflexia. The neurological exam at presentation and throughout the clinical course is used to assess the presence or absence of UMN and LMN signs in each of the 4 body regions (cranial, cervical, thoracic and lumbosacral). Any objective evidence of sensory loss, autonomic insufficiency, ocular motility disturbance, tremor or extrapyramidal motor abnormality (as in Parkinson's disease) is considered to exclude the diagnosis of classic MND [6-8]. Cognitive dysfunction or dementia has, until recently, also been considered to exclude the diagnosis of MND $[9,10]$. New developments have completely changed our understanding of this phenomenon and have broadened our understanding of the spectrum of neurodegeneration in MND.

The MND known as ALS is characterized by the progressive loss of both UMNs and LMNs. Progressive muscular atrophy (PMA) is the form of MND characterized by the progressive loss of LMNs. Not infrequently, patients initially presenting with PMA may develop UMN signs later in the disease course. If so, the PMA designation is changed to LMN-onset ALS. Importantly, PMA patients who never develop any clinical UMN signs on neurological examination may have UMN pathology at autopsy. Primary lateral sclerosis (PLS) is the form of MND characterized by the progressive loss of UMNs. Similar to PMA, patients who initially present with PLS may develop LMN signs later in their disease course. If so, the PLS designation is changed to UMN-onset ALS. As with PMA, PLS patients with no clinical LMN signs may have LMN pathology on autopsy. Progressive bulbar palsy is an UMN and/or LMN disorder that remains restricted to muscles of the cranial or bulbar segment. Very frequently, however, patients who initially present in this way later develop UMN and/or LMN abnormalities in other body segments. If so, the progressive bulbar palsy designation is changed to bulbar-onset ALS $[1,4$, $11,12]$.

ALS is the most common adult MND. It has a yearly incidence (new cases in the population) of 2-3 per 100,000 population that is considered to be unaffected by racial or ethnic variables. Below the age of 65 the incidence of ALS is 2 to 1 male compared to female; above this age the incidence is gender neutral. From the time of diagnosis the average life expectancy is 3-4 years. The most common cause of death is hypercarbic respiratory failure produced by progressive weakness of respiratory musculature. Weakness of the swallowing musculature may produce malnutrition and dehydration that may also prove fatal $[1,11,12]$.

There is no cure for MND and palliation with a focus upon physical, emotional and psychosocial needs of the patient is the cornerstone of clinical care from the time of diagnosis to death. A single drug, riluzole, has demonstrated modest slowing of disease progression in the ALS form of MND. Given overlapping clinical and pathologic features, this drug is usually offered to individuals affected by the other forms as well [13-16].

\section{Pattern of Cognitive Abnormalities in MND}

It is now clear that MND is clinically and pathologically linked to the disorder known as FTLD. About 5$10 \%$ of individuals with MND will develop a dementing illness resembling FTLD. The clinical features of FTLD may precede or follow the development of motor abnormalities in MND. FTLD is among the most common of the cortical dementing illnesses, and is almost as common as Alzheimer's disease among individuals less than 65 years of age, with a prevalence of 3.6-15.0 per 100,000, and an average age of onset of about 60 [17]. The clinical manifestations of FTLD are predicted on the basis of the frontal and temporal cortical predominance of pathology. These include a progressive form of aphasia, or disorder of social comportment and executive functioning. There are 3 defined phenotypes of FTLD, categorized on the basis of clinical profiles evident at presentation [18]. The behavioral or frontal variant, known as frontotemporal dementia (FTD), most commonly presents as a disorder of social comportment with personality change, disinhibition, lack of empathy and amotivation. Dysexecutive features include inattention, poor planning, and impaired abstraction and problem solving. Accompanying abnormalities often include a decline in personal hygiene, mental rigidity and inflexibility, hyperorality with 
Table 1. Demographic characteristics and results of cognitive screening in a consecutive cohort of 227 patients with MND

\begin{tabular}{|c|c|c|c|c|c|c|}
\hline & \multicolumn{6}{|l|}{ Onset site } \\
\hline & bulbar & cervical & thoracic & lumbosacral & respiratory & cognitive \\
\hline Patients, $\mathrm{n}$ & $45(19.8 \%)$ & $62(27.3 \%)$ & $8(3.5 \%)$ & $104(45.8 \%)$ & $3(1.3 \%)$ & $5(2.2 \%)$ \\
\hline Male & $33.3 \%$ & $74.2 \%$ & $62.5 \%$ & $42.3 \%$ & $100.0 \%$ & $20.0 \%$ \\
\hline Mean age at symptom onset, years & $59.7(13.2)$ & $56.4(15.7)$ & $58.5(11.7)$ & $58.1(13.5)$ & $70.9(5.5)$ & $59.8(9.0)$ \\
\hline Mean age at diagnosis, years & $61.8(11.6)$ & $57.3(18.1)$ & $59.5(11.5)$ & $61.0(11.8)$ & $72.3(5.5)$ & $63.8(9.0)$ \\
\hline Mean survival, days ${ }^{1}$ & $1,215(46.9)$ & $1,029(60.5)$ & $400(25.5)$ & $2,076(351.8)$ & $\mathrm{n} / \mathrm{a}$ & $\mathrm{n} / \mathrm{a}$ \\
\hline Patients with abnormal verbal fluency, $\mathrm{n}$ & $13(28.9 \%)$ & $12(19.4 \%)$ & $5(62.5 \%)$ & $33(31.8 \%)$ & $2(66.7 \%)$ & $2(40.0 \%)$ \\
\hline Patients with abnormal frontal behavioral inventory, $\mathrm{n}$ & $11(24.4 \%)$ & $12(19.4 \%)$ & $2(25.0 \%)$ & $29(27.9 \%)$ & $1(33.3 \%)$ & $3(60.0 \%)$ \\
\hline $\begin{array}{l}\text { Patients with abnormal verbal fluency and } \\
\text { frontal behavioral inventory, } \mathrm{n}\end{array}$ & $3(6$. & $3(4.8 \%)$ & $2(25.0 \%)$ & $12(11.5 \%)$ & 0 & $2(40.0 \%)$ \\
\hline
\end{tabular}

Figures in parentheses are standard deviations, unless indicated otherwise. $\mathrm{n} / \mathrm{a}=$ Insufficient data to calculate.

${ }^{1}$ For deceased patients, days from symptom onset.

carbohydrate craving, perseverative and stereotyped behavior, and changes in speech output with echolalia, perseveration and eventually mutism. Patients with progressive nonfluent aphasia exhibit effortful speech with agrammatism, paraphasic errors and anomia. Oral apraxia, impaired repetition, alexia and agraphia also may be present, though language comprehension and word meaning is preserved in the early stage. This is associated with anterior peri-Sylvian disease in the left hemisphere. In contrast, speech in semantic dementia is fluent but often impoverished in content. There is impaired word meaning that interferes with naming, comprehension and reading, and eventually loss of object knowledge emerges. This is associated with left anterior and ventral temporal disease. Memory and praxis are relatively well preserved across phenotypes.

Patients with MND who are cognitively abnormal may manifest features of any of these syndromes, and MND patients meeting the Neary criteria for all 3 subtypes of FTLD have been reported [19]. The most common cognitive abnormality detected in patients with MND is impaired verbal fluency [20,21]. This is typically assessed through a timed word generation task wherein patients are given $1 \mathrm{~min}$ to produce a list of words that either begin with a target letter (for example ' $F$ ') or name members of a semantic category (for example 'animals'). Abnormalities of verbal fluency predict that detailed neuropsychiatric testing will confirm a diagnosis of FTLD as defined by the Neary criteria [10]. This task is not sensitive to behavioral impairments, so a proportion of MND patients with normal word generation who undergo additional neuropsychiatric evaluation will also meet criteria for FTLD, most commonly of the FTD subtype [10].

Motor Neuron Disease and

Neurosignals 2008;16:85-90

\section{Prevalence of Cognitive Abnormalities in MND}

There is a much larger group of MND patients who do not have a frank dementia but nevertheless have some cognitive limitations. Screening tests may detect cognitive deficits in $30-50 \%$ of patients with MND [10, 19, 2225]. To account for the observation that a small proportion of patients will have frank dementia while a somewhat higher proportion will show abnormalities on cognitive testing, a continuum for cognitive abnormalities in patients with MND has been proposed. This continuum includes: frankly demented patients who meet clinical criteria for FTLD and can also be classified as having FTD, progressive nonfluent aphasia or semantic dementia; patients with attenuated social/behavioral abnormalities or cognitive abnormalities (for example executive dysfunction or language abnormalities); cognitively normal individuals $[19,26]$. Research is ongoing to determine the prevalence of each of these conditions.

\section{The University of Pennsylvania Experience}

The formal diagnosis of cognitive impairment in MND would ideally be accomplished through detailed neuropsychiatric testing of all patients with MND. Given time constraints, a quick screen is of practical utility. In order to capture the 2 major clinical aspects of FTLD, we have been applying a cognitive screen to all patients seen at our multidisciplinary ALS Association Center at the University of Pennsylvania. The screen includes a verbal fluency task and the Frontal Behavioral Inventory (FBI). The FBI is a 24 -item questionnaire that is completed by the caregiver and quantifies features seen in FTD, allow- 


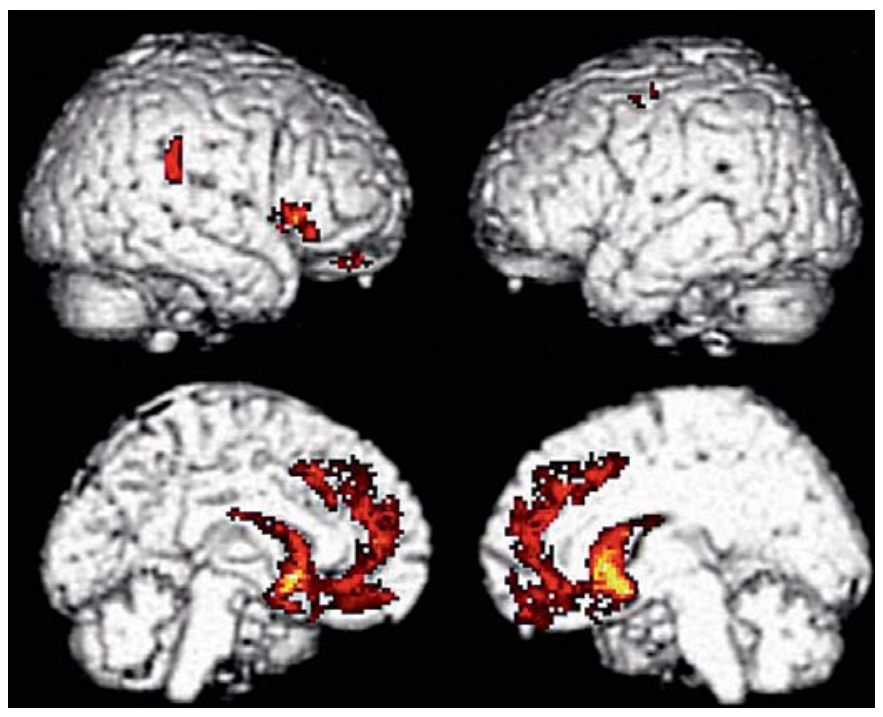

Fig. 1. Left/right medial and left/right lateral views demonstrating gray matter atrophy in 6 patients with MND/FTLD as compared to age-matched controls.

ing the distinction of FTD from other dementias [27]. The verbal fluency task is administered orally or in writing, depending on the patient's preference. A cutoff score equivalent to 2 standard deviations worse than performance of age- and education-matched controls is used to identify individuals at risk for cognitive and behavioral abnormalities in association with the clinical diagnosis of MND.

Over the course of 6 months, 227 patients with MND were evaluated at our institution. Table 1 summarizes the demographic and clinical features of these patients. Onset was bulbar in $19.8 \%$, respiratory in $1.3 \%$, cognitive in $2.2 \%$ and $\mathrm{limb} /$ thoracic in the remaining $76.7 \%$. The pattern of clinical onset in MND is important to note because FTLD may occur more commonly in the bulbaronset form of MND, and may occur much less commonly in the PMA form of MND. Verbal fluency testing was performed on 172 patients (75.8\%). The FBI was administered to 69 patients (30.4\%). Verbal fluency was abnormal at least once in $39.0 \%$ of those tested. The FBI was abnormal at least once in $84.1 \%$ of those tested. Abnormalities on both measures were seen in $31.9 \%$ of those tested. This number comports well with the experience of other large centers investigating cognitive disorders in MND [10]. Among patients with bulbar onset, $28.9 \%$ demonstrated abnormalities of verbal fluency, $24.4 \%$ scored abnormally on the FBI and $6.7 \%$ were abnormal on both measures. For patients with limb, thoracic or re- spiratory onset, the corresponding numbers were 29.4, 24.9 and $9.6 \%$ with impairments on both cognitive and social measures. These data are not consistent with the notion that cognitive/behavioral abnormalities are more common in bulbar-onset disease. These preliminary observations do not, however, address the question of whether cognitive/behavioral abnormalities are more common in patients who manifest bulbar dysfunction as part of their clinical syndrome at some point during the course of their disease.

\section{Neuroimaging in Motor Neuron Disease with Cognitive Impairment}

Neuroimaging is carried out during the usual evaluation of patients with suspected MND, largely to exclude alternative diagnoses. The findings on brain magnetic resonance imaging (MRI) are most commonly normal, though hyperintensity in the corticospinal tracts may occasionally be seen on T2 and FLAIR sequences and hypointensity of the motor strip on $\mathrm{T} 1$ sequences has also been reported [28, 29].

Voxel-based morphometry (VBM) uses T1-weighted MRI images to assess the whole brain and identify regional structural atrophy quantitatively, based on operator-independent volumetric analysis. VBM is a particularly attractive technique to use in patients with MND and cognitive impairment, as the entire brain is analyzed quantitatively, including nonmotor regions that may be compromised in MND. VBM has identified global brain atrophy as well as regional atrophy in the motor cortex, left medial frontal gyrus [30], and pre- and postcentral gyrus [31] in nondemented MND patients. Gray matter atrophy in frontotemporal regions has also been seen in MND patients with clinical FTLD [32] as well as milder cognitive impairment [33]. We have recently used VBM to compare patients with MND/FTLD and age-matched controls. This is illustrated in figure 1. Significant differences were found in the bilateral medial frontal, left middle frontal, right orbital frontal, right inferior frontal, right hippocampus and right inferior parietal regions.

Other MRI modalities are used on a research basis. Diffusion tensor imaging is a structural neuroimaging technique that assigns numerical values to the cohesiveness of white matter tracts in the brain through measurement of the magnitude and directionality of water flow $[34,35]$. This technique has shown early promise for use in the posterior limb of the internal capsule of patients with MND [36-38] and for examining white matter tracts 
in patients with FTLD [39]. We have successfully applied this technique to measure the volume of the corticospinal tract in patients with MND, and we find that this is reduced when compared to controls [40].

Magnetic resonance spectroscopy (MRS) assesses brain metabolites that reflect neuronal integrity. The motor cortex is the usual region of interest for MRS in MND patients, and there is much data to suggest that neuronal integrity is measurably altered in this area $[28,41-48]$. We too have found that MRS of the precentral gyrus is abnormal in patients with MND, and that this predicts disease duration [36].

\section{Pathophysiology of MND and FTLD}

Much work has been devoted to the investigation of superoxide dysmutase in MND [49, 50]. More recently, investigators have pursued a pathophysiologic mechanism more closely linked to the commonly observed pathology of MND, most notably the ubiquitin-immunoreactive, tau-negative neuronal inclusions. This pathology is identical to the single most common form of pathology found in FTLD, known as FTLD with ubiquitin-positive but tau-negative inclusions (FTLD-U) [51]. The ubiquitinated protein in both FTLD-U and MND conditions was recently identified as TDP-43 [52]. This discovery is extraordinarily important for several reasons. Not only does this provide an important new target for drug discovery in the treatment of these conditions, but the common occurrence of TDP-43 pathology in both MND and FTLD provides a crucial biological rationale for the recent clinical observation of cognitive difficulties in MND. Indeed, several different conformations of TDP-43 have been identified with immunostaining, and MND and
FTD are both observed to have several of these conformational subtypes. Ongoing work is investigating the biological significance of these conformational changes.

\section{Conclusions}

MND and FTLD appear to overlap at several levels. Clinically, MND is no longer thought to be a purely motor disorder, and a large number of MND patients also appear to share clinical features of FTLD. A modest number of cases with MND also display a full-blown FTLD syndrome manifesting as primary progressive aphasia or a disorder of social comportment and executive functioning. A much larger number of individuals with MND - as many as half the cases of MND in some series - exhibit an attenuated pattern of cognitive, language and social difficulty. The presence of these cognitive and social impairments has important consequences for ongoing medical care and end-of-life planning. This clinical overlap is also seen in biomarkers. For example, imaging studies show cortical atrophy in nonmotor regions of the frontal and temporal lobes, consistent with the cognitive and social difficulties seen in many of these patients. Diffusion tensor imaging and spectroscopic studies likewise show important changes and motor and nonmotor regions consistent with the clinical features shared by MND and FTLD. Finally, the presence of TDP-43 in the ubiquinated histopathologic inclusions of both MND and FTLD emphasizes the shared biological basis for these disorders.

\section{Acknowledgment}

This work was supported in part by AG17586.

\section{References}

1 Rowland LP, Shneider NA: Amyotrophic lateral sclerosis. N Engl J Med 2001;344:16881700.

2 Rowland LP: How amyotrophic lateral sclerosis got its name: the clinical-pathologic genius of Jean-Martin Charcot. Arch Neurol 2001;58:512-515.

3 Rowland LP: What's in a name? Amyotrophic lateral sclerosis, motor neuron disease, and allelic heterogeneity. Ann Neurol 1998;43:691-694.

4 Rowland LP: Diagnosis of amyotrophic lateral sclerosis. J Neurol Sci 1998;160:S6-S24.
5 Rowland LP: Amyotrophic lateral sclerosis. Curr Opin Neurol 1994;7:310-315.

-6 Beghi E, Balzarini C, Bogliun G, et al: Reliability of the El Escorial diagnostic criteria for amyotrophic lateral sclerosis. Neuroepidemiology 2002;21:265-270.

7 Forbes RB, Colville S, Swingler RJ: Are the El Escorial and Revised El Escorial criteria for ALS reproducible? A study of inter-observer agreement. Amyotroph Lateral Scler Other Motor Neuron Disord 2001;2:135-138.
-8 Belsh JM: ALS diagnostic criteria of El Escorial Revisited: Do they meet the needs of clinicians as well as researchers? Amyotroph Lateral Scler Other Motor Neuron Disord 2000;1(suppl 1):S57-S60.

9 Lomen-Hoerth C: Characterization of amyotrophic lateral sclerosis and frontotemporal dementia. Dement Geriatr Cogn Disord 2004;17:337-341.

10 Lomen-Hoerth C, Murphy J, Langmore S, Kramer JH, Olney RK, Miller B: Are amyotrophic lateral sclerosis patients cognitively normal? Neurology 2003;60:1094-1097. 
11 Mitsumoto H, Przedborski S, Gordon PH: Amyotrophic Lateral Sclerosis. New York, Taylor and Francis, 2006.

12 Rowland LP: Primary lateral sclerosis: disease, syndrome, both or neither? J Neurol Sci 1999;170:1-4.

13 Miller ET: Caring versus curing. Rehabil Nurs 2006;31:182-183.

14 Miller RG, Mitchell JD, Lyon M, Moore DH: Riluzole for amyotrophic lateral sclerosis (ALS)/motor neuron disease (MND). Amyotroph Lateral Scler Other Motor Neuron Disord 2003;4:191-206.

15 Miller RG: Examining the evidence about treatment in ALS/MND. Amyotroph Lateral Scler Other Motor Neuron Disord 2001;2: 3-7.

16 Miller RG, Rosenberg JA, Gelinas DF, et al: Practice parameter: The care of the patient with amyotrophic lateral sclerosis (an evidence-based review): report of the Quality Standards Subcommittee of the American Academy Of Neurology: ALS Practice Parameters Task Force. Neurology 1999;52: 1311-1323.

17 Rosso SM, Donker Kaat L, Baks T, et al: Frontotemporal dementia in the netherlands: patient characteristics and prevalence estimates from a population-based study. Brain 2003;126:2016-2022.

18 Neary D, Snowden JS, Gustafson L, et al: Frontotemporal lobar degeneration: a consensus on clinical diagnostic criteria. Neurology 1998;51:1546-1554.

19 Murphy JM, Henry RG, Langmore S, Kramer JH, Miller BL, Lomen-Hoerth C: Continuum of frontal lobe impairment in amyotrophic lateral sclerosis. Arch Neurol 2007; 64:530-534.

20 Abrahams S, Leigh PN, Harvey A, Vythelingum GN, Grise D, Goldstein LH: Verbal fluency and executive dysfunction in amyotrophic lateral sclerosis (ALS). Neuropsychologia 2000;38:734-747.

21 Bak TH, Hodges JR: The effects of motor neurone disease on language: further evidence. Brain Lang 2004;89:354-361.

22 Rakowicz WP, Hodges JR: Dementia and aphasia in motor neuron disease: an underrecognised association? J Neurol Neurosurg Psychiatry 1998;65:881-889.

-23 Massman PJ, Sims J, Cooke N, Haverkamp LJ, Appel V, Appel SH: Prevalence and correlates of neuropsychological deficits in amyotrophic lateral sclerosis. J Neurol Neurosurg Psychiatry 1996;61:450-455.

24 Rippon GA, Scarmeas N, Gordon PH, et al: An observational study of cognitive impairment in amyotrophic lateral sclerosis. Arch Neurol 2006;63:345-352.

25 Ringholz GM, Appel SH, Bradshaw M, Cooke NA, Mosnik DM, Schulz PE: Prevalence and patterns of cognitive impairment in sporadic ALS. Neurology 2005;65:586590.
26 Murphy J, Henry R, Lomen-Hoerth C: Establishing subtypes of the continuum of frontal lobe impairment in amyotrophic lateral sclerosis. Arch Neurol 2007;64:330334.

27 Kertesz A, Nadkarni N, Davidson W, Thomas AW: The Frontal Behavioral Inventory in the differential diagnosis of frontotemporal dementia. J Int Neuropsychol Soc 2000;6: 460-468.

28 Chan S, Shungu DC, Douglas-Akinwande A, Lange DJ, Rowland LP: Motor neuron diseases: comparison of single-voxel proton $\mathrm{MR}$ spectroscopy of the motor cortex with MR imaging of the brain. Radiology 1999;212: 763-769.

29 Oba H, Araki T, Ohtomo K, et al: Amyotrophic lateral sclerosis: T2 shortening in motor cortex at MR imaging. Radiology 1993;189:843-846.

30 Kassubek J, Unrath A, Huppertz HJ, et al: Global brain atrophy and corticospinal tract alterations in ALS, as investigated by voxelbased morphometry of 3-D MRI. Amyotroph Lateral Scler Other Motor Neuron Disord 2005;6:213-220.

31 Grosskreutz J, Kaufmann J, Fradrich J, Dengler R, Heinze HJ, Peschel T: Widespread sensorimotor and frontal cortical atrophy in amyotrophic lateral sclerosis. BMC Neurol 2006;6:17.

32 Chang JL, Lomen-Hoerth C, Murphy J, et al: A voxel-based morphometry study of patterns of brain atrophy in ALS and ALS/ FTLD. Neurology 2005;65:75-80.

33 Mezzapesa DM, Ceccarelli A, Dicuonzo F, et al: Whole-brain and regional brain atrophy in amyotrophic lateral sclerosis. Am J Neuroradiol 2007;28:255-259.

34 Pierpaoli C, Basser PJ: Toward a quantitative assessment of diffusion anisotropy. Magn Reson Med 1996;36:893-906.

35 Basser PJ, Pajevic S, Pierpaoli C, Duda J, Aldroubi A: In vivo fiber tractography using DT-MRI data. Magn Reson Med 2000;44: 625-632.

36 Wang S, Poptani H, Woo JH, et al: Amyotrophic lateral sclerosis: diffusion-tensor and chemical shift MR imaging at 3.0 T. Radiology 2006;239:831-838.

37 Ellis CM, Simmons A, Dawson JM, Williams SC, Leigh PN: Distinct hyperintense MRI signal changes in the corticospinal tracts of a patient with motor neuron disease. Amyotroph Lateral Scler Other Motor Neuron Disord 1999;1:41-44.

38 Toosy AT, Werring DJ, Orrell RW, et al: Diffusion tensor imaging detects corticospinal tract involvement at multiple levels in amyotrophic lateral sclerosis. J Neurol Neurosurg Psychiatry 2003;74:1250-1257.
39 Studholme C, Cardenas V, Blumenfeld R, et al: Deformation tensor morphometry of semantic dementia with quantitative validation. Neuroimage 2004;21:1387-1398.

40 Wang S, Poptani H, Bilello M, et al: Diffusion tensor imaging in amyotrophic lateral sclerosis: volumetric analysis of the corticospinal tract. Am J Neuroradiol 2006;27:12341238.

41 Kaufmann P, Pullman SL, Shungu DC, et al: Objective tests for upper motor neuron involvement in amyotrophic lateral sclerosis (ALS). Neurology 2004;62:1753-1757.

42 Jones AP, Gunawardena WJ, Coutinho CM, Gatt JA, Shaw IC, Mitchell JD: Preliminary results of proton magnetic resonance spectroscopy in motor neurone disease (amytrophic lateral sclerosis). J Neurol Sci 1995;129: 85-89.

43 Abe K, Takanashi M, Watanabe Y, et al: Decrease in $\mathrm{N}$-acetylaspartate/creatine ratio in the motor area and the frontal lobe in amyotrophic lateral sclerosis. Neuroradiology 2001;43:537-541.

-44 Mitsumoto H, Ulug AM, Pullman SL, et al: Quantitative objective markers for upper and lower motor neuron dysfunction in ALS. Neurology 2007;68:1402-1410.

45 Pohl C, Block W, Traber F, et al: Proton magnetic resonance spectroscopy and transcranial magnetic stimulation for the detection of upper motor neuron degeneration in ALS patients. J Neurol Sci 2001;190:21-27.

46 Pohl C, Block W, Karitzky J, et al: Proton magnetic resonance spectroscopy of the motor cortex in 70 patients with amyotrophic lateral sclerosis. Arch Neurol 2001;58:729735.

-47 Rooney WD, Miller RG, Gelinas D, Schuff N, Maudsley AA, Weiner MW: Decreased Nacetylaspartate in motor cortex and corticospinal tract in ALS. Neurology 1998;50: 1800-1805.

48 Bowen BC, Bradley WG: Amyotrophic lateral sclerosis: the search for a spectroscopic marker of upper motoneuron involvement. Arch Neurol 2001;58:714-716.

49 Beckman JS, Estevez AG, Crow JP, Barbeito L: Superoxide dismutase and the death of motoneurons in ALS. Trends Neurosci 2001; 24:S15-S20.

50 Morrison BM, Morrison JH: Amyotrophic lateral sclerosis associated with mutations in superoxide dismutase: a putative mechanism of degeneration. Brain Res Brain Res Rev 1999;29:121-135.

51 Forman MS, Farmer J, Johnson JK, et al: Frontotemporal dementia: clinicopathological correlations. Ann Neurol 2006;59:952962.

52 Neumann M, Sampathu DM, Kwong LK, et al: Ubiquitinated TDP-43 in frontotemporal lobar degeneration and amyotrophic lateral sclerosis. Science 2006;314:130-133. 